\title{
THE CALKIN ALGEBRA IS NOT COUNTABLY HOMOGENEOUS
}

\author{
ILIJAS FARAH AND ILAN HIRSHBERG
}

\begin{abstract}
We show that the Calkin algebra is not countably homogeneous, in the sense of continuous model theory. We furthermore show that the connected component of the unitary group of the Calkin algebra is not countably homogeneous.
\end{abstract}

Motivated by their study of extensions of $\mathrm{C}^{*}$-algebras, Brown, Douglas and Fillmore asked whether the Calkin algebra has a $K$-theory reversing automorphism and whether it has outer automorphisms at all (4, Remark 1.6 (ii)]). By [16] and [8] the answer to the latter question is independent from ZFC. In particular, since inner automorphisms fix $K$-theory, a negative answer to the former question is relatively consistent with ZFC. It is not known whether the existence of a $K$-theory reversing automorphism of the Calkin algebra is relatively consistent with ZFC. All known automorphisms of the Calkin algebra ([16] and [8, §1]) act trivially on its $K$-theory, as they are implemented by a unitary on every separable subalgebra of the Calkin algebra.

A scenario for using Continuum Hypothesis to construct a $K$-theory reversing automorphism of the Calkin algebra on separable Hilbert space, denoted $Q$, was sketched in [11, §6.3] and in [9, §7.1]. The following theorems demonstrate that this strategy does not work and suggest that question of the existence of such automorphism is even more difficult than previously thought (for terminology see below and [1] or [10]).

Theorem 1. The Calkin algebra $Q$ is not countably homogeneous, and this is witnessed by a quantifier-free type.

Theorem 2. The group $U_{0}(Q)$ of Fredholm index zero unitaries in $Q$ is not countably homogeneous.

Our theorems give negative answers to [10, Questions 5.2 and 5.7] and a novel obstruction to countable saturation of $Q$. In [10, Question 5.1] it was asked whether all obstructions to (quantifier-free) countable saturation of $Q$ are of $K$-theoretic nature. The obstruction given in our results is finer than the Fredholm index, but it is $K$-homological and therefore ultimately $K$-theoretical. In addition, the obstruction given in Theorem 1 is quantifier-free and one given in Theorem 2 appears to have little to do with the Fredholm index. It should be noted that one of the key ideas, using $\operatorname{Ext}\left(M_{2 \infty}\right)$, is due to N.C. Phillips, and it was already used in the proof of [10, Proposition 4.2].

Date: September 22, 2021. 
Model theory of $\mathrm{C}^{*}$-algebras and their unitary groups is based on [2] and described in [11, $§ 2.3 .1$ and $\S 2.3 .3$, respectively]. Formulas in logic of metric structures are defined recursively. In case of $\mathrm{C}^{*}$-algebras, atomic formulas are expressions of the form $\|t(\bar{x})\|$ where $t$ is a noncommutative ${ }^{*}$-polynomial in variables $\bar{x}=\left(x_{1}, \ldots, x_{n}\right)$. The set of all formulas is the smallest set $\mathbb{F}$ containing all atomic formulas such that (i) for every $n$, all continuous $f:[0, \infty)^{n} \rightarrow[0, \infty)$ and all $\phi_{1}, \ldots, \phi_{n}$ in $\mathbb{F}$ the expression $f\left(\phi_{1}, \ldots, \phi_{n}\right)$ belongs to $\mathbb{F}$ and (ii) if $\phi \in \mathbb{F}, m \geq 1$, and $x$ is a variable symbol than both $\sup _{\|x\| \leq m} \phi$ and $\inf _{\|x\| \leq m} \phi$ belong to $\mathbb{F}$ (see [11, §2.4]). If $\phi\left(x_{1}, \ldots, x_{n}\right.$ ) is a formula, $A$ is a $C^{*}$-algebra, and $a_{1}, \ldots, a_{n}$ are elements of $A$, then the interpretation $\phi\left(a_{1}, \ldots, a_{n}\right)^{A}$ is obviously defined by recursion. A condition is any expression of the form $\phi \leq r$ for formula $\phi$ and $r \geq 0$ and type is a set of conditions ([11, §4.3]). As every expression of the form $\phi=r$ is equivalent to the condition $\max (\phi, r) \leq r$ and every expression of the form $\phi \geq r$ is equivalent to the condition $\min (0, r-\phi) \leq 0$, we shall freely refer to such expressions as conditions. For $n \geq 1$, the $n$-type is a type such that free variables occurring in its conditions are included in $\left\{x_{1}, \ldots, x_{n}\right\}$. It is important that each free variable $x$ is associated with a domain of quantification, which in our case reduces to asserting that $\|x\| \leq m$ for some fixed $m$.

Given a $\mathrm{C}^{*}$-algebra $A$ and sequence $\bar{a}=\left(a_{j}: n \in \mathbb{N}\right)$ in $A$, the type of $\bar{a}$ in $A$ is the set of all conditions $\phi\left(x_{1}, \ldots, x_{m}\right) \leq r$ such that $\phi\left(a_{1}, \ldots, a_{m}\right)^{A} \leq r$. A structure $C$ is said to be countably homogeneous if for every two sequences $\bar{a}=\left(a_{n}: n \in \mathbb{N}\right)$ and $\bar{b}=\left(b_{n}: n \in \mathbb{N}\right)$ with the same type and every $c \in C$ there exists $d \in C$ such that $(\bar{a}, c)$ and $(\bar{b}, d)$ have the same type. Our proof of the failure of countable homogeneity in $Q$ will show that sequences $\bar{a}$ and $\bar{b}$ can be chosen to be finite.

We recall the definitions the semigroups $\operatorname{Ext}(A)$ and $\operatorname{Ext}^{w}(A)$. If $A$ is a unital $\mathrm{C}^{*}$-algebra, we consider injective unital *-homomorphisms $\pi: A \rightarrow Q$ (such a $*_{-}$ homomorphism is the Busby invariant of an extension of $A$ by the compacts). By slight abuse of notation, we call such a *-homomorphism an extension. Two extensions $\pi_{j}: A \rightarrow Q$, for $j=1,2$ are said to be weakly equivalent if there is a unitary $u$ in the Calkin algebra such that $\pi_{1}=\operatorname{Ad} u \circ \pi_{2}$. If $u$ above is furthermore required to have Fredholm index zero then we say that these extensions are equivalent. The set of such *-homomorphisms is equipped with the direct sum operation (using implicitly the fact that $\left.M_{2}(Q) \cong Q\right)$, and the set of equivalence relations forms a semigroup, denoted $\operatorname{Ext}^{w}(A)$ or $\operatorname{Ext}(A)$, respectively. They correspond to semigroups $\operatorname{Ext}_{*}^{u}(A, \mathcal{K})$ where $*=s, w$ and $\mathcal{K}$ denotes the algebra of compact operators on separable Hilbert space as defined in [3, Definition 15.6.3, Proposition 15.6.2 and $\S 15.4$ (2), (3)] (see also [4, 12, 1]).

If $A$ is a simple unital $\mathrm{C}^{*}$-subalgebra of $Q$ and $p \in A^{\prime} \cap Q$ is a nonzero projection, then $a \mapsto p a p$ is an injective unital *-homomorphism from $A$ into $p Q p \cong Q$. The isomorphism between $p Q p$ and $Q$ used here is chosen by picking an isometry $v$ such that $v v^{*}=p$, and the map $Q \rightarrow p Q p$ is given by $x \mapsto v x v^{*}$. The choice of $v$ is unique up to multiplication by a unitary, and therefore it does not affect the Ext $^{w}$ class. (The 
choice of $v$ can affect the Ext class, and therefore the choice of $p$ only determines the weak equivalence class.) Therefore projections in $A^{\prime} \cap Q$ determine Ext ${ }^{w}$-classses of unital extensions of $A$, after identifying $p Q p$ with $Q$ in the manner we described.

A subalgebra $A$ of $Q$ is split if there is a unital ${ }^{*}$-homomorphism from $\Phi: A \rightarrow B(H)$ such that $\pi \circ \Phi=\mathrm{id}_{A}$. The following lemma is related to [12, Lemma 5.1.2 and Lemma 5.1.2].

Lemma 3. Let $A$ be a simple separable unital subalgebra of $Q$ and let $p$ and $q$ be projections in $A^{\prime} \cap Q$. Then $p$ and $q$ are Murray-von Neumann equivalent in $A^{\prime} \cap Q$ if and only if the extensions of $A$ corresponding to $p$ and $q$ are weakly equivalent.

Proof. The direct implication is trivially true because of our convention that for nonzero $p \in A^{\prime} \cap Q$ we identify $p Q p$ with $Q$ and $p$ with unital extension $a \mapsto p a p$ of $A$. We now prove the converse implication. If the extensions corresponding to $p$ and $q$ are weakly equivalent, then there exists a partial isometry $v$ in $Q$ such that $v^{*} v=p, v v^{*}=q$, and $v p a p v^{*}=q a q$ for all $a \in A$. It will suffice to check that $v \in A^{\prime} \cap Q$. Fix $a \in A$. We have $v a v^{*}=v v^{*} a v v^{*}$, and since $v v^{*} \in A^{\prime} \cap Q$, we have $v v^{*} a v v^{*}=a v v^{*}$ and therefore $v a v^{*}=a v v^{*}$. Multiplying by $v$ on the right hand side and using $v^{*} v \in A^{\prime} \cap Q$ we have $v v^{*} v a=a v v^{*} v$. But since $v$ is a partial isometry we have $v v^{*} v=v$, thus showing that $v a=a v$.

If $A$ is a separable, unital and nuclear $\mathrm{C}^{*}$-algebra then $\operatorname{Ext}^{w}(A)$ is a group ([7, p. 586]). This implies that every extension of $A$ corresponds to some $p \in A^{\prime} \cap Q$. To see that, if $\pi_{1}: A \rightarrow Q$ is any given extension, then there exists an extension $\pi_{2}: A \rightarrow Q$ such that $\pi_{1} \oplus \pi_{2}$ is weakly equivalent to $\operatorname{id}_{A}$. The extension $\pi_{1}$ corresponds to the projection $\left(\begin{array}{ll}1 & 0 \\ 0 & 0\end{array}\right) \in\left(\pi_{1} \oplus \pi_{2}\right)(A)^{\prime} \cap M_{2}(Q)$. When we identify $\pi_{1} \oplus \pi_{2}$ with id $\operatorname{id}_{A}$ via a unitary and an isomorphism $M_{2}(Q) \cong Q$, the above matrix is identified with a projection $p \in A^{\prime} \cap Q$ as required.

Lemma 4. Let $A$ be a separable unital subalgebra of $Q$ such that $\operatorname{Ext}^{w}(A)$ is a group. Then the Cuntz algebra $\mathcal{O}_{2}$ unitally embeds into $A^{\prime} \cap Q$ if and only if $A$ is split.

Proof. Assume first that $A$ is split. Recall that by Voiculescu's theorem ([18], [1, Section 4]), all trivial extensions of $A$ are equivalent. In particular, $\operatorname{id}_{A}$ is equivalent to $\mathrm{id}_{A} \oplus \mathrm{id}_{A}: A \rightarrow Q \otimes M_{2}$. Thus, if $A$ is split then there is a projection $p \in A^{\prime} \cap Q$ such that both $p A p$ and $(1-p) A(1-p)$ are split in $p Q p$ and $(1-p) Q(1-p)$, respectively. Lemma 3 implies that $1, p$ and $1-p$ are Murray-von Neumann equivalent and therefore $\mathcal{O}_{2}$ embeds unitally into $A^{\prime} \cap Q$.

Now assume $\mathcal{O}_{2}$ unitally embeds into $A^{\prime} \cap Q$. Then the extension of $A$ corresponding to 1 is an idempotent in $\operatorname{Ext}(A)$. Since the identity is the only idempotent in a group, $A$ is split.

Let $A$ denote the CAR algebra, $M_{2}$. $A$ is singly generated by [13]. Fix a generator $g$ for $A$. Since $A$ is nuclear, $\operatorname{Ext}^{w}(A)$ is a group. 
Lemma 5. For every unital extension $\pi$ of $A$, the type of $\pi(g)$ in $Q$ is the same as the type of $\pi_{0}(g)$ corresponding to the trivial extension $\pi_{0}$ of $A$.

Proof. Represent $A$ as a direct limit of $M_{2^{n}}(\mathbb{C})$ and choose $a_{n} \in M_{2^{n}}(\mathbb{C})$ such that $\lim _{n} a_{n}=g$. Fix a unital extension $\pi$ of $A$. For $n \in \mathbb{N}$ the group $\operatorname{Ext}^{w}\left(M_{2^{n}}(\mathbb{C})\right)$ is trivial, and therefore the type of $\pi\left(a_{n}\right)$ in $Q$ is the same as the type of $\pi_{0}\left(a_{n}\right)$ in $Q$. Fix a formula $\phi(x)$. Since the interpretation $b \mapsto \phi(b)^{Q}$ is continuous, we have

$$
\phi(\pi(g))^{Q}=\lim _{n} \phi\left(\pi\left(a_{n}\right)\right)^{Q}=\lim _{n} \phi\left(\pi_{0}\left(a_{n}\right)\right)^{Q}=\phi\left(\pi_{0}(g)\right)^{Q} .
$$

Since $\phi$ was arbitrary, the conclusion follows.

Proof of Theorem 1. Fix a unital *-homomorphism $\pi$ of $A$ into $Q$ and consider the 2-type in $x_{1}, x_{2}$ consisting of conditions

$$
x_{j} \pi(g)=\pi(g) x_{j}, \quad x_{j}^{*} x_{j}=1, \quad x_{1} x_{1}^{*}+x_{2} x_{2}^{*}=1
$$

for $j=1,2$. By Lemma 4, this type is realized if and only if $\pi$ is the trivial extension.

Since $A$ has both trivial and nontrivial extensions (as a matter of fact, $\operatorname{Ext}^{w}(A)$ is uncountable by [5, Proposition 3] or [17]) and the type of $\pi(g)$ does not depend on the choice of the extension $\pi$ by Lemma 5, $Q$ is not (countably) homogeneous.

The salient point in our proof of Theorem 2 is the fact that the presence of $\mathcal{O}_{2}$ in $A^{\prime} \cap Q$ can be detected from $A^{\prime} \cap U_{0}(Q)$. We note that in [14, Theorem 4.6] it was shown that if $B$ and $C$ are simple $\mathrm{C}^{*}$-algebras such that their unitary groups are isometrically isomorphic then this isomorphism extends to an isomorphism or an anti-isomorphism of $B$ and $C$. We were not able, however, to use this result directly.

By Voiculescu's theorem ([18]) for a unital separable $\mathrm{C}^{*}$-subalgebra $A$ of $Q$ one has $\left(A^{\prime} \cap Q\right)^{\prime}=A$ and $Z\left(A^{\prime} \cap Q\right)=Z(A)$. We need the following self-strengthening of this result.

Lemma 6. If $A$ is a unital separable $\mathrm{C}^{*}$-subalgebra of $Q$ then $\left(A^{\prime} \cap U_{0}(Q)\right)^{\prime}=A$ and $Z\left(A^{\prime} \cap U_{0}(Q)\right)=Z(A) \cap U_{0}(Q)$.

Proof. Assume $b \in Q$ is such that $b \notin A$. Since $A=\left(A^{\prime} \cap Q\right)^{\prime}$, there exists an element $x \in A^{\prime} \cap Q$ such that $x b \neq b x$. By replacing $x$ by its real or imaginary part, we may assume that $x$ is self-adjoint, and we may assume that $\|x\|<\pi$. Set $u=\exp (i x)$. Then $u \in A^{\prime} \cap U_{0}(Q)$ and since $x \in \mathrm{C}^{*}(u)$, we have $u b \neq b u$. Therefore $b \notin\left(A^{\prime} \cap U_{0}(Q)\right)^{\prime}$. Since $A=\left(A^{\prime} \cap Q\right)^{\prime}$ and $b$ was arbitrary, this proves $\left(A^{\prime} \cap U_{0}(Q)\right)^{\prime}=$ A.

The second equality is a standard consequence of the first. If $b \in Z\left(A^{\prime} \cap U_{0}(Q)\right)$, then by the above $b \in A$ and therefore $b \in Z(A)$. Since $Z(A) \subseteq A^{\prime} \cap Q$, the conclusion follows.

Lemma 7. The Cuntz algebra $\mathcal{O}_{2}$ is the universal $\mathrm{C}^{*}$-algebra generated by three unitaries $u, v$ and $w$ satisfying the following relations:

(1) $u^{2}=v^{3}=w^{6}=1$. 
(2) $\|w-1\|=1$.

(3) $u w^{3} u=-w^{3}$.

(4) $v w^{2} v^{2}=e^{2 \pi i / 3} w^{2}$.

Proof. In [6, Theorem 2.6] Choi proved that every $\mathrm{C}^{*}$-algebra generated by unitaries $u$ and $v$ and projection $p$ satisfying the following conditions is isomorphic to $\mathcal{O}_{2}$ :

(5) $u^{2}=v^{3}=1$

(6) $p+u p u=1$ and $p+v p v^{2}+v^{2} p v=1$.

Denote $\gamma=e^{2 \pi i / 6}$. It is straightforward to check that such $u$ and $v$, together with

$$
w=p+\gamma v p v^{2}+\gamma^{5} v^{2} p v
$$

satisfy our conditions. It will therefore suffice to prove that our conditions imply

(7) $w=p+\gamma q+\gamma^{5} r$, with projections $p, q$, $r$ satisfying $p+q+r=1$.

(8) $u p u+p=1$ and $p+v p v^{2}+v^{2} p v=1$.

Since $w^{6}=1$ and $\|w-1\|=1=\left|e^{2 \pi i / 6}-1\right|, \operatorname{Sp}(w)$ is contained in $\left\{\gamma, 1, \gamma^{5}\right\}$, with at least one of $\gamma$ or $\gamma^{5}$ belonging to it. By (4), the unitaries $w^{2}$ and $\gamma^{2} w^{2}$ are conjugate and $\operatorname{Sp}\left(w^{2}\right)=\left\{\gamma^{2} \lambda: \lambda \in \operatorname{Sp}\left(w^{2}\right)\right\}$. Therefore $\operatorname{Sp}(w)=\left\{\gamma, 1, \gamma^{5}\right\}$, and we can write

(9) $w=p+\gamma q+\gamma^{5} r$

for projections $p, q, r$ satisfying $p+q+r=1$. By applying (44) to (9) we obtain $v p v^{2}=q$ and $v q v^{2}=r$. In particular, $p+v p v^{2}+v^{2} p v=1$.

Since $u w^{3} u=-w^{3}, u$ and $w^{3}$ generate a unital copy of $M_{2}(\mathbb{C})$ and $p=\left(w^{3}+1\right) / 2$ (the equality follows by (9)) is a projection such that $p+u p u=1$.

Thus $u, v$ and $p=\left(w^{3}+1\right) / 2$ satisfy Choi's conditions and the proof is complete.

Lemma 8. There is a 4-type $\mathbf{s}(\bar{x})$ in the language of metric groups such that for a unital $\mathrm{C}^{*}$-algebra $A$ and a closed group $G$ satisfying $U_{0}(A) \subseteq G \subseteq A$ and $Z(G)=\mathbb{T}$ the following are equivalent.

(1) $\mathbf{s}$ is realized in $G$.

(2) A has a unital subalgebra isomorphic to $\mathcal{O}_{2}$ whose unitary group is included in $G$.

Proof. Define a type $\mathbf{s}(\bar{x})$ consisting of the following conditions:

(3) $x_{1}^{2}=x_{2}^{3}=x_{3}^{6}=1$.

(4) $\sup _{y}\left\|x_{4} y x_{4}^{-1} y^{-1}-1\right\|=0$

(5) $\left\|x_{4}-1\right\|=1$

(6) $\left\|x_{3}-1\right\|=1$.

(7) $x_{1} x_{3}^{3} x_{1}=-x_{3}^{3}$.

(8) $x_{2} x_{3}^{2} x_{2}^{2}=x_{4}^{2} x_{3}^{2}$.

Observe that $x_{4}$ satisfies condition (4) if and only if $x_{4} \in Z(G)$. Write $\gamma=e^{2 \pi i / 6}$, and note that $\gamma$ and $\gamma^{5}$ are the only elements of $Z(A)=\mathbb{T}$ at the distance exactly 1 from the identity. Therefore (5) implies that $x_{4}=\gamma \cdot 1$ or $x_{4}=\gamma^{5} \cdot 1$. 
If $x_{4}=\gamma \cdot 1$, the remaining conditions are satisfied by $x_{1}, x_{2}$ and $x_{3}$ in $G$ if and only if they satisfy the assumptions of Lemma 7. If $x_{4}=\gamma^{5} \cdot 1$, then $x_{1}, x_{2}$ and $x_{3}^{-1}$ satisfy those conditions. Either way, we see that if $G$ realizes the type then by Lemma 7 there exists a unital copy of $\mathcal{O}_{2}$ in $A$.

Since every unitary in $\mathcal{O}_{2}$ is of the form $\exp (i a)$ for a self-adjoint $a$ ([15]), its unitary group is connected. Therefore if $A$ and $G$ are as above then $A$ has a unital copy of $\mathcal{O}_{2}$ if and only if it has a unital copy of $\mathcal{O}_{2}$ whose unitary group is included in $G$. By the above, this is equivalent to $\mathbf{s}$ being realized in $G$.

Proof of Theorem Q 2 . As in the proof of Theorem 1, let $A$ denote the CAR algebra $M_{2^{\infty}}$ and let $g_{j}$, for $j \in \mathbb{N}$, be an enumeration of a dense subgroup of the unitary group $U(A)$. Fix a unital ${ }^{*}$-homomorphism $\pi: A \rightarrow Q$. Since the unitary group of $A$ is connected, we have $\pi(U(A)) \subseteq U_{0}(Q)$ and $\pi(A)^{\prime} \cap U_{0}(Q)=U(\pi(A))^{\prime} \cap U_{0}(Q)$.

As in Theorem 1, the type of $\left(\pi\left(g_{j}\right): j \in \omega\right)$ does not depend on the choice of $\pi$. Since $Z(A)=\mathbb{C}$, Lemma 6] implies $Z\left(\pi(A)^{\prime} \cap U_{0}(Q)\right)=\mathbb{T}$.

Let 4-type $\mathbf{s}^{+}(\bar{x})$ consist of $\mathbf{s}(\bar{x})$ as in Lemma 8 together with all conditions of the form

$$
\left\|g_{j} x_{k} g_{j}^{-1} x_{k}^{-1}-1\right\|=0
$$

for $j \in \mathbb{N}$ and $1 \leq k \leq 3$. Then $\mathbf{s}^{+}$is realized in $U_{0}(Q)$ if and only if $\mathbf{s}$ is realized in $U(\pi(A))^{\prime} \cap U_{0}(Q)=\pi(A)^{\prime} \cap U_{0}(Q)$. Since the assumptions of Lemma 8 are satisfied, $\mathbf{s}$ is realized in $U(\pi(A))^{\prime} \cap U_{0}(Q)$ if and only if $\mathcal{O}_{2}$ unitally embeds into $\pi(A)^{\prime} \cap Q$. Since there are $\pi_{1}: A \rightarrow Q$ and $\pi_{2}: A \rightarrow Q$ such that $\pi_{1}(A)^{\prime} \cap Q$ has a unital copy of $\mathcal{O}_{2}$ and $\pi_{2}(A)^{\prime} \cap Q$ does not, our proof is complete.

We conclude with a remark on the role of the Continuum Hypothesis in the construction of a possible $K$-theory reversing automorphism of the Calkin algebra. Woodin's $\sum_{1}^{2}$ absoluteness theorem (see [19]) implies that, under a suitable large cardinal assumption, the following holds. If there exists a forcing extension in which the Calkin algebra has a $K$-theory reversing automorphism, then every forcing extension in which the Continuum Hypothesis holds contains a $K$-theory reversing automorphism of the Calkin algebra. This means that if the existence of a $K$-theory reversing automorphism of the Calkin algebra is consistent with ZFC, then it most likely follows from the Continuum Hypothesis.

Acknowledgment. The results of this note were proved during first author's visit to the Ben Gurion University in May 2015. He would like to thank the Department of Mathematics, and the second author in particular, for their warm hospitality. We would also like to thank the referee for suggesting several improvements.

\section{REFERENCES}

1. W. Arveson, Notes on extensions of $C^{*}$-algebras, Duke Math. J. 44 (1977), no. 2, 329-355. 
2. I. Ben Yaacov, A. Berenstein, C.W. Henson, and A. Usvyatsov, Model theory for metric structures, Model Theory with Applications to Algebra and Analysis, Vol. II (Z. Chatzidakis et al., eds.), London Math. Soc. Lecture Notes Series, no. 350, Cambridge University Press, 2008, pp. 315-427.

3. B. Blackadar, K-theory for operator algebras, second ed., Mathematical Sciences Research Institute Publications, vol. 5, Cambridge University Press, Cambridge, 1998.

4. L. G. Brown, R. G. Douglas, and P. A. Fillmore, Extensions of $C^{*}$-algebras and $K$-homology, Ann. of Math. (2) 105 (1977), no. 2, 265-324.

5. S. J. Cho, Strong extensions vs. weak extensions of $C^{*}$-algebras, Canad. Math. Bull. 21 (1978), no. $2,143-147$.

6. M.-D. Choi, A simple $C^{*}$-algebra generated by two finite-order unitaries, Canad. J. Math. 31 (1979), no. 4, 867-880.

7. M.-D. Choi and E.G. Effros, The completely positive lifting problem for c $^{*}$-algebras, Annals of Mathematics (1976), 585-609.

8. I. Farah, All automorphisms of the Calkin algebra are inner, Ann. of Math. (2) 173 (2011), no. $2,619-661$.

9. __ Logic and operator algebras, Proceedings of the Seoul ICM (S.Y. Jang, Y.R. Kim, D.-W. Lee, and I. Yie, eds.), vol. II, Kyung Moon SA, 2014, pp. 15-40.

10. I. Farah and B. Hart, Countable saturation of corona algebras, C.R. Math. Rep. Acad. Sci. Canada 35 (2013), 35-56.

11. I. Farah, B. Hart, and D. Sherman, Model theory of operator algebras II: Model theory, Israel J. Math. 201 (2014), 477-505.

12. N. Higson and J. Roe, Analytic K-homology, Oxford Mathematical Monographs, Oxford University Press, Oxford, 2000, Oxford Science Publications.

13. C.L. Olsen and W.R. Zame, Some $C^{*}$-algebras with a single generator, Trans. Amer. Math. Soc. 215 (1976), 205-217.

14. A.L.T. Paterson, Harmonic analysis on unitary groups, J. Funct. Anal. 53 (1983), no. 3, 203223.

15. N.C. Phillips, Exponential length and traces, Proc. Roy. Soc. Edinburgh Sect. A 125 (1995), no. $1,13-29$.

16. N.C. Phillips and N. Weaver, The Calkin algebra has outer automorphisms, Duke Math. J. 139 (2007), no. 1, 185-202.

17. M. Pimsner and S. Popa, On the Ext-group of an AF-algebra, Rev. Roumaine Math. Pures Appl. 23 (1978), no. 2, 251-267.

18. D. Voiculescu, A non-commutative Weyl-von Neumann theorem, Rev. Roumaine Math. Pures Appl. 21 (1976), no. 1, 97-113.

19. W.H. Woodin, Beyond $\sum_{1}^{2}$ absoluteness, Proceedings of the International Congress of Mathematicians, Vol. I (Beijing, 2002), Higher Ed. Press, Beijing, 2002, pp. 515-524.

Department of Mathematics and Statistics, York University, 4700 Keele Street, North York, Ontario, Canada, M3J 1P3

E-mail address: ifarah@yorku.ca

$U R L:$ http://www.math.yorku.ca/ ifarah

Department of Mathematics, Ben Gurion University of the Negev, P.O.B. 653, BE'ER, SHEVA 84105, IsRAEL

E-mail address: ilan@math.bgu.ac.il

URL: http://www.math.bgu.ac.il/ ilan/ 